\title{
Immunoregulation and genotype in colorectal cancer
}

\author{
Sarah E Church*, Gabriela Bindea, Bernhard Mlecnik, Tessa Fredricksen, Jerome Galon \\ From 30th Annual Meeting and Associated Programs of the Society for Immunotherapy of Cancer (SITC 2015) \\ National Harbor, MD, USA. 4-8 November 2015
}

Classical methods for determining malignant disease prognosis are based upon characterizing the morphology and location of tumor cells. While this analysis provides important information about a patient's disease it fails to capture the biological complexity of the tumor microenvironment and the contribution of the anti-tumor immune response. Basic histological quantification of $\mathrm{T}$ lymphocyte density, cytotoxicity, and memory by CD3, $\mathrm{CD} 8$, and CD45RO, respectively, has demonstrated that increased infiltration of $\mathrm{T}$ lymphocytes is associated with statistically significant improvement in patients' diseasefree survival (DFS) and overall survival (OS) in colorectal carcinoma (CRC). Delineating the location of T lymphocytes into two areas within the primary tumor, the center and the invading margin is termed Immunoscore.

In this study we examined whether tumor genotype could predict the clinical outcome of patients with CRC and if combining Immunoscore with analysis of the tumor genotype could further enhance the accuracy of predicting clinical outcome. We analyzed 200 primary colorectal tumor samples for mutations in 50 common driver genes, including those implicated in models of colon carcinogenesis (APC, KRAS, BRAF, SMAD4). Each mutation was then assessed for prediction of DFS. Patients presented with a mean of $4 \pm 2$ mutations. The most common mutations were in KRAS (58\%), TP53 (49\%) and APC (37\%). Neither, a single mutation or the number of mutations significantly predicted clinical outcome.

We examined whether mutations in the primary tumor were associated with presence of metastasis. Mutational profiles were remarkably similar between primary tumors from patients whether or not they had metastatic progression at the time of surgery. None of the 50 major cancer genes was more frequently mutated in patients with metastasis. However, using integrative analysis we found

INSERM, Paris, France original work is properly cited. The Creative Commons Public Domain Dedication waiver (http://creativecommons.org/publicdomain/ zero/1.0/) applies to the data made available in this article, unless otherwise stated. 